\title{
A Vector Geometry-Based Eddy Detection Algorithm and Its Application to a High-Resolution Numerical Model Product and High-Frequency Radar Surface Velocities in the Southern California Bight
}

\author{
FRANCESCO NENCIOLI \\ Ocean Physics Laboratory, University of California, Santa Barbara, Santa Barbara, California \\ ChANGMing DONG \\ IGPP, University of California, Los Angeles, Los Angeles, California \\ TOMMY DICKEY \\ Ocean Physics Laboratory, University of California, Santa Barbara, Santa Barbara, California \\ LIBE WASHBURN \\ ICESS, University of California, Santa Barbara, Santa Barbara, California \\ JAMES C. MCWiLLiams \\ IGPP, University of California, Los Angeles, Los Angeles, California
}

(Manuscript received 16 July 2009, in final form 19 October 2009)

\begin{abstract}
Automated eddy detection methods are fundamental tools to analyze eddy activity from the large datasets derived from satellite measurements and numerical model simulations. Existing methods are either based on the distribution of physical parameters usually computed from velocity derivatives or on the geometry of velocity streamlines around minima or maxima of sea level anomaly. A new algorithm was developed based exclusively on the geometry of the velocity vectors. Four constraints characterizing the spatial distribution of the velocity vectors around eddy centers were derived from the general features associated with velocity fields in the presence of eddies. The grid points in the domain for which these four constraints are satisfied are detected as eddy centers. Eddy sizes are computed from closed contours of the streamfunction field, and eddy tracks are retrieved by comparing the distribution of eddy centers at successive time steps. The results were validated against manually derived eddy fields. Two parameters in the algorithm can be modified by the users to optimize its performance. The algorithm is applied to both a high-resolution model product and highfrequency radar surface velocity fields in the Southern California Bight.
\end{abstract}

\section{Introduction}

Mesoscale eddies are ubiquitous features in the world's oceans, and they play a major role in ocean circulation as well as in heat and mass transport (e.g., McWilliams 2008). They can have a profound influence on biological productivity, upper ocean ecology and biogeochemistry,

Corresponding author address: Francesco Nencioli, Ocean Physics Laboratory, Department of Geography, University of California, Santa Barbara, Santa Barbara, CA 93106.

E-mail: francesco.nencioli@opl.ucsb.edu and thus in elemental cycling and fluxes (i.e., Falkowski et al. 1991; McGillicuddy et al. 1998; McNeil et al. 1999; Benitez-Nelson et al. 2007). In the past decade, several studies have focused on the statistical characterization of mesoscale eddy activity within specific regions through the analysis of satellite measurements or results from numerical models (i.e., Isern-Fontanet et al. 2003; Morrow et al. 2004; Chelton et al. 2007; Chaigneau et al. 2008; Doglioli et al. 2007).

A suitable definition of an eddy and the implementation of an algorithm to automatically identify and track mesoscale and submesoscale features are fundamental 
to study eddy activity from large datasets. A few methods have been proposed, based either on the physical or geometrical characteristics of the flow field. Methods based on physical characteristics identify eddies using the values of a specified parameter that exceed a chosen threshold; methods based on geometrical characteristics of the flow field identify eddies based on the shape or curvature of the instantaneous streamlines (Sadarjoen and Post 2000). Therefore, automated eddy detection algorithms can be categorized into three types: 1) physical parameter based; 2) flow geometry based; and 3) hybrid, which involves both physical parameters and flow geometry characteristics.

The study by McWilliams (1990) represents one of the earliest works in automated eddy detection. The algorithm was developed to quantitatively measure the properties specific of coherent vortices from a numerical solution of decaying two-dimensional (2D) turbulence. Based on the notion that rotation dominates within a vortex, relative vorticity $\xi$ is the physical parameter used for detection. Eddy centers are identified by local minima and maxima of $\xi$, and eddy boundaries are defined by the points around the center where $\xi / \xi_{\text {cen }}<0.2\left(\xi_{\text {cen }}\right.$ is the center value). A series of constraints is applied to the geometrical characteristics of the detected structures, and only the structures that do not depart excessively from axisymmetry are considered representative of a vortex. For this reason, this method belongs to the last category.

In the category of eddy detection algorithms based on physical parameters, one of the most widely used is based on the properties of the Okubo-Weiss parameter $W$ (Okubo 1970; Weiss 1991). This parameter is computed from the horizontal velocity field as $W=s_{\mathrm{sh}}^{2}+s_{\mathrm{st}}^{2}-\xi^{2}$, where $s_{\mathrm{sh}}$ and $s_{\mathrm{st}}$ are the shear and strain deformation, respectively, and $\xi$ is the vertical component of vorticity. Here, $W$ quantifies the relative importance of deformation with respect to rotation. Because the velocity field within a vortex is dominated by rotation, ocean eddies are generally characterized by negative values of $W$. For this reason, it is possible to identify these features by closed contours of $W=W_{0}$, where $W_{0}$ is a chosen negative threshold value. Despite this method being frequently used to detect eddies from sea level altimetry (SLA) data (i.e., Isern-Fontanet et al. 2003; Morrow et al. 2004; Chelton et al. 2007), the velocity derivatives induce extra noise in the $W$ field. This is usually reduced by applying a smoothing algorithm, which, however, might also remove physical information. A few studies have evidenced some limitations of this method (Sadarjoen and Post 2000; Chaigneau et al. 2008): a tendency toward excess of eddy detection (i.e., identifying a feature as an eddy when it is not) seems to persist even after smoothing the $W$ field; furthermore, when long time series are an- alyzed, the value of the threshold parameter $W_{0}$ needs to be continuously adjusted according to the varying eddy properties of the velocity field.

Another method that belongs to the first category is the one proposed by Doglioli et al. (2007). As in the method proposed by McWilliams (1990), the physical parameter used for eddy detection is $\xi$. To highlight eddy features, a two-dimensional wavelet analysis is applied to the $\xi$ field: relative vorticity is first expanded into wavelet bases, and then a smoothed field is reconstructed using only the wavelets with the largest coefficients. Vortices can be identified by the connected regions where the reconstructed $\xi \neq 0$. Sometimes smallscale filaments can be detected as vortices. To minimize this error, filaments are automatically excluded from the analysis by imposing a constraint on the minimum width of a detected structure.

A different approach is adopted in the winding-angle method proposed by Sadarjoen and Post (2000), which belongs to the second category. It was developed under the assumption that vortices can be defined as features characterized by roughly circular or spiral instantaneous streamlines around their cores (Robinson 1991). As a first step, instantaneous streamlines are derived from the velocity field; then, the cumulative change of direction of the segments that compose a given streamline (winding angle) is computed for each streamline. Eddies are identified by streamlines with a winding angle $|\alpha| \geq 2 \pi$, which corresponds to a closed or spiral curve.

This method was used by Chaigneau et al. (2008) to analyze eddy activity in the eastern South Pacific from SLA data. Comparison with the results obtained with the Okubo-Weiss method on the same dataset showed that the winding-angle method has a higher chance of successfully detecting mesoscale eddies and most importantly a much smaller excess of detection error. The price for this is paid in terms of more expensive computational requirements (Sadarjoen and Post 2000). Chaigneau et al. (2008) found a way around this problem by applying the method only within those regions of the domain where vortices were identified by local maxima and minima of SLA. For this reason, their method can be classified as hybrid (third category): a physical quantity (SLA) is used to identify the eddies, and then the geometrical characteristics of the flow field (streamlines) are used to define eddy boundaries.

In this study, we present a new method for the automated detection and tracking of ocean eddies. This method falls into the second category because it is entirely based on the geometry of the flow field. The algorithm was developed to investigate mesoscale and submesoscale eddy activity in the Southern California Bight (SCB) from the results of a 1-km-resolution numerical experiment 


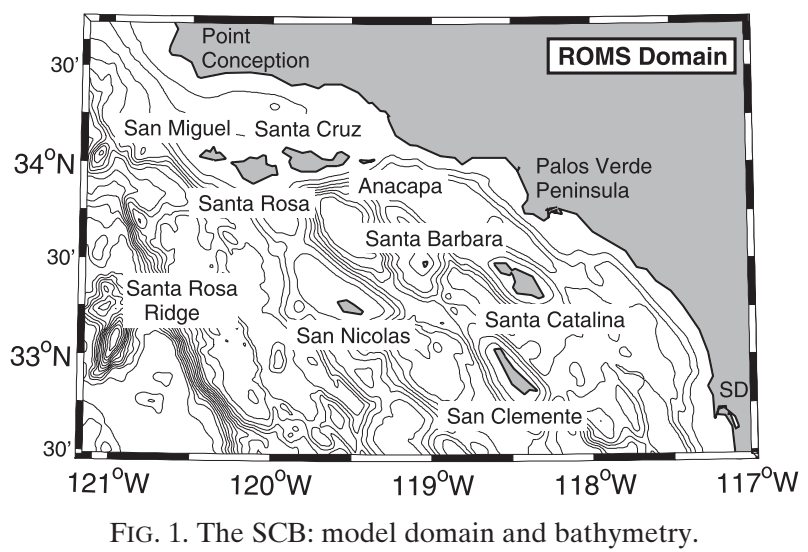

using the Regional Ocean Modeling System (ROMS) [for interested readers, the detection algorithm presented in this paper is freely available upon request to the author (it consists of a set of routines in MatLab)].

The SCB extends from Point Conception to San Diego, and it is bounded to the west by the Santa Rosa Ridge (Fig. 1). This region is characterized by the presence of eight islands: the islands of San Miguel, Santa Rosa, Santa Cruz, and Anacapa (called the Channel Islands) form the outer limit of the Santa Barbara Channel, south of Point Conception; Santa Catalina Island is located south of Palos Verdes Peninsula; and San Nicolas, Santa Barbara, and San Clemente Islands are farther offshore, to the south and to the west. Previous studies based on in situ and remote sensing data (Caldeira et al. 2005), as well as on numerical modeling (Dong and McWilliams 2007), have shown that because of the presence of the islands, the SCB is characterized by an intense eddy activity. This region is therefore ideal for developing and testing our new detection method.

With the support of direct observations from field experiments (i.e., Nencioli et al. 2008), we have derived a series of constraints that allow us to identify vortices directly from the characteristics of the velocity field. The algorithm is proposed as an alternative to other existing methods, particularly for the analysis of eddy activity from the results of high-resolution numerical experiments. The velocity field from these types of experiments is more "complex" than from satellite altimetry or from numerical simulations at lower resolutions because highresolution models can reproduce small processes down to the submesoscale. Furthermore, in the SCB domain, the presence of the islands has a big impact on the relative vorticity field. As shown by Dong and McWilliams (2007), $\xi$ increases both close to the islands and in the islands' wakes, mainly because of lateral stress and current shear. Preliminary results using the Okubo-Weiss method (not shown) revealed that strong frontal regions as well as shear regions in the island wakes are often associated with values of $W$ similar to or lower than eddy features, making it hard to choose threshold values capable of distinguishing between the two. Another important consideration is that, in our case, the velocity field is not directly derived from the SLA field; therefore, not all the eddies are characterized by an SLA minimum or maximum. For these reasons, we decided to develop an algorithm in which the eddy detection is completely independent from parameters derived using velocity derivatives as well as from the SLA field.

This paper presents an accurate description of both detection and tracking methodologies, and particular attention is dedicated to the validation of the detection algorithm. After eddies are detected, several physical parameters can be computed, and spatial and temporal statistics can be derived. However, a complete characterization of the eddy activity in the SCB from the 8 years of high-resolution ROMS simulation is beyond the scope of this paper and will be presented by C. Dong et al. (2010, unpublished manuscript). The algorithm can be used to detect eddies from any velocity field. Here, we briefly present the results from the application to highfrequency (HF) radar velocities in the Santa Barbara Channel (Emery et al. 2004). HF radars are widely used to measure surface ocean currents close to the coastline. An algorithm capable of automatically detecting mesoscale and submesoscale vortices from these recorded velocity fields would represent an important tool for the analysis of eddy activity in the nearshore region.

The paper is organized as follows: section 2 gives a brief description of the numerical model and the HF radar dataset; section 3 describes in detail the method used for eddy detection and tracking and discusses the results from the algorithm validation; section 4 shows preliminary results from the application of the algorithm to HF radar datasets; and final conclusions are presented in section 5 .

\section{Data}

\section{a. Ocean model}

The algorithm was developed to analyze eddy activity from a high-resolution numerical model product from Dong et al. (2009a) and Dong and McWilliams (2007). ROMS, which solves the rotating primitive equations (Shchepetkin and McWilliams 2005), was used to generate daily velocity fields for 8 years, from 1996 to 2003 . The model domain covers the whole SCB region with a horizontal grid spacing of $1 \mathrm{~km}(258 \times 386$ grid points $)$ and 40 vertical levels. The fine resolution allows all of the eight islands in the SCB to be represented in the domain. The model is forced at the surface by reanalyzed meteorological 
fluxes from the fifth-generation Pennsylvania State University-National Center for Atmospheric Research (PSU-NCAR) Mesoscale Model (MM5; Conil and Hall 2006). Global oceanic data [Simple Oceanic Data Assimilation (SODA)] are used for boundary conditions at the four open boundaries (Carton et al. 2000). The model results have been extensively assessed against observational data, both in situ and remote sensed. The comparisons show that the model is able to reproduce realistic conditions, including both seasonal and interannual fluctuations. The eddy-scale dynamics are also well resolved in the product. For more details about the model configuration and the model validation, please refer to Dong et al. (2009a). ROMS uses a C grid and vertical S coordinates. Both meridional $u$ and zonal $v$ components of velocity were interpolated on the same grid at 5-m depth before the algorithm was applied.

\section{b. HF radar dataset}

Since 1997, near-surface currents in the Santa Barbara Channel have been measured using an array of HF radars (SeaSondes, manufactured by CODAR Ocean Sensors, Mountain View, California) along the mainland coast and on Santa Cruz Island. The number of radars operating in the channel has increased from two to six since measurements began. The HF radars used in this study operate at $12-13 \mathrm{MHz}$ and measure currents over the upper $1 \mathrm{~m}$ of the water column. The radars measure surface currents using the Doppler shift associated with Bragg scattering of radio waves from surface gravity waves with wavelengths equal to half the transmitted wavelength $(\approx 24 \mathrm{~m})$. The radial components of current velocity are measured every $10 \mathrm{~min}$, and the spatial resolution of radial currents is $1.5 \mathrm{~km}$ in range and $5^{\circ}$ in azimuth. Surface current vectors are interpolated onto a 2-km square grid based on all radial currents within $3 \mathrm{~km}$ of each grid point using a least squares fit. Emery et al. (2004) discuss data processing procedures and performance of the HF radar array in more detail.

\section{Eddy detection and tracking}

\section{a. Detection algorithm}

An eddy can be intuitively defined as a region in which the velocity field exhibits a rotary flow: that is, a region in which the velocity vectors rotate clockwise or counterclockwise around a center. This definition is consistent with the ones assumed for the Okubo-Weiss and the $\xi$ methods (McWilliams 1990; Doglioli et al. 2007), where a vortex is defined as a region where rotation dominates, as well as with the definition assumed for the windingangle method, where, following Robinson (1991), a vor- tex is defined as a region where instantaneous streamlines spiral around a center.

In the past two decades, several studies have shown that eddy velocity fields are generally characterized by some typical features (e.g., Olson 1991; Dickey et al. 2008), such as minimum velocities in the proximity of the eddy center and tangential velocities that increase approximately linearly with distance from the center before reaching a maximum value and then decaying. A recent study of a mesoscale cyclone (Cyclone Opal) sampled on the lee of the Big Island of Hawaii showed that these features can be successfully used to estimate the location of an eddy's center from its acoustic Doppler current profiler-based velocity field (Nencioli et al. 2008).

The eddy detecting algorithm developed for this study is based on similar concepts. Four constraints were derived in conformance with the eddy velocity field definition and characteristics described above. Eddy centers are determined at the points where all of the constraints are satisfied. The four constraints follow:

(i) along an east-west (EW) section, $v$ has to reverse in sign across the eddy center and its magnitude has to increase away from it;

(ii) along a north-south (NS) section, $u$ has to reverse in sign across the eddy center and its magnitude has to increase away from it: the sense of rotation has to be the same as for $v$;

(iii) velocity magnitude has a local minimum at the eddy center; and

(iv) around the eddy center, the directions of the velocity vectors have to change with a constant sense of rotation and the directions of two neighboring velocity vectors have to lay within the same or two adjacent quadrants (the four quadrants are defined by the north-south and west-east axes: the first quadrant encompasses all the directions from east to north, the second quadrant encompasses the directions from north to west, the third quadrant encompasses the directions from west to south, and the fourth quadrant encompasses the directions from south to east).

The constraints require two parameters to be specified: one for the first, second, and fourth constraints and one for the third one. The first parameter, $a$, defines how many grid points away the increases in magnitude of $v$ along the EW axes and $u$ along the NS axes are checked. It also defines the curve around the eddy center along which the change in direction of the velocity vectors is inspected. The second parameter, $b$, defines the dimension (in grid points) of the area used to define the local minimum of velocity. These two parameters give flexibility to the algorithm: they set the minimum size of the 


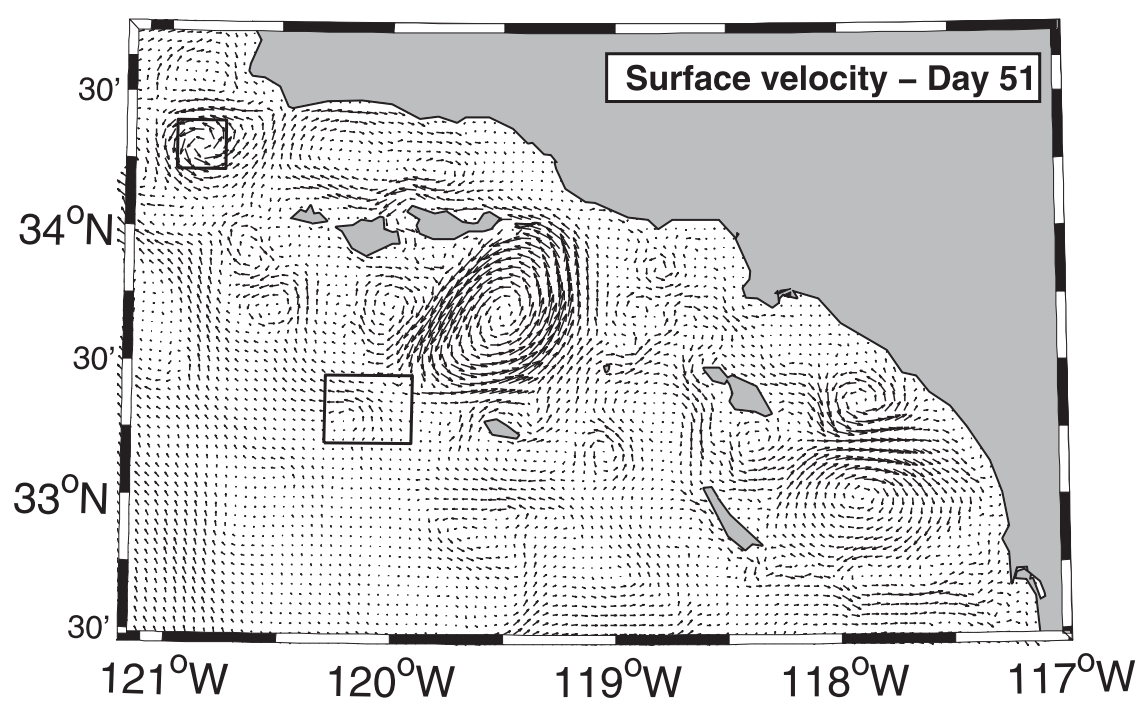

FIG. 2. Surface velocity field for day 51 (20 Feb 1996). Vectors are plotted every four grid points. The eddy within the box in the top-left corner is used as an example in Fig. 3 to show how the four constraints are applied. Velocity vectors within the larger box are plotted in Fig. 4.

detectable vortices and allow the algorithm to be applied to grids with different resolution (i.e., Dong et al. 2009b). On the other hand, their values need to be accurately tuned depending on the characteristics and spatial resolution of a given dataset in order to optimize the algorithm performance. Success and excess of detection can significantly vary, and few sensitivity tests had to be performed in order to specify optimal values of those two parameters. For eddy detection from a high-resolution ROMS simulation, we used $a=4$ and $b=3$. Motivations for these choices are given in section $3 \mathrm{~b}$, where results from sensitivity experiments are discussed.

In the paragraphs below we will use the vortex within the box in the top-left corner of Fig. 2 as an example to describe the algorithm in more detail and to better visualize how the four constraints are used to identify eddy centers. Starting from the first row of the surface velocity matrix, latitudinal sections (east-west) of $v$ are analyzed, looking for contiguous points of opposite sign. An example is represented by the dashed line on Fig. 3a; the two black dots along the section indicate contiguous points with opposite values of the $v$ component. For each pair, the algorithm analyzes sign and magnitude of $v$ at $a=4$ points away ( $4 \mathrm{~km}$ for the present model resolution). The two crosses indicate such points relative to the pair along the dashed line in Fig. 3a. Following the first constraint, only the pairs for which $v$ is still opposite in sign but higher in magnitude are considered indicative of the presence of a vortex and thus retained in the analysis (the circles in Fig. 3a). From the east-west variation of $v$, it is already possible to determine the sense of rotation of the eddy: if $v$ changes from negative to positive (progressing from the east toward the west) as the center is crossed, then the rotation is anticyclonic; if it changes from positive to negative, then it is cyclonic (Northern Hemisphere; it is the opposite in the Southern Hemisphere).

For the next step, the second constraint is applied to the longitudinal sections (north-south) of $u$ across the points for which the first constraint was satisfied. From the pairs in Fig. 3a, we take as an example the one indicated by the two dots in Fig. 3b. Values of $u$ at $a=4$ points away northward and southward are inspected (indicated by the two crosses in Fig. 3b). The magnitude of $u$ at those points has to be larger than at the starting point, whereas its sign has to be consistent with the sense of rotation determined from the longitudinal variation of $v$ for the same point: that is, $u$ has to change from negative to positive (progress from south to north) if the rotation is anticyclonic and from positive to negative if it is cyclonic (again, Northern Hemisphere; the opposite in the Southern Hemisphere). The circles in Fig. 3b indicate the points among the ones in Fig. 3a that also satisfy the second constraint. Being based on the variation of the two velocity components along different directions, the first two constraints are independent from each other. Therefore, the points for which both are satisfied are independent from the order with which the two are applied.

The third constraint is applied to the points for which both constraints on $v$ and $u$ are satisfied, and it requires the presence of a local minimum of velocity close to them. If an eddy is present, these points are usually close to the velocity minimum (as can be seen in Fig. 3b) and therefore the searching area can be small. It is defined by moving $\pm b$ points away from a given point along both 


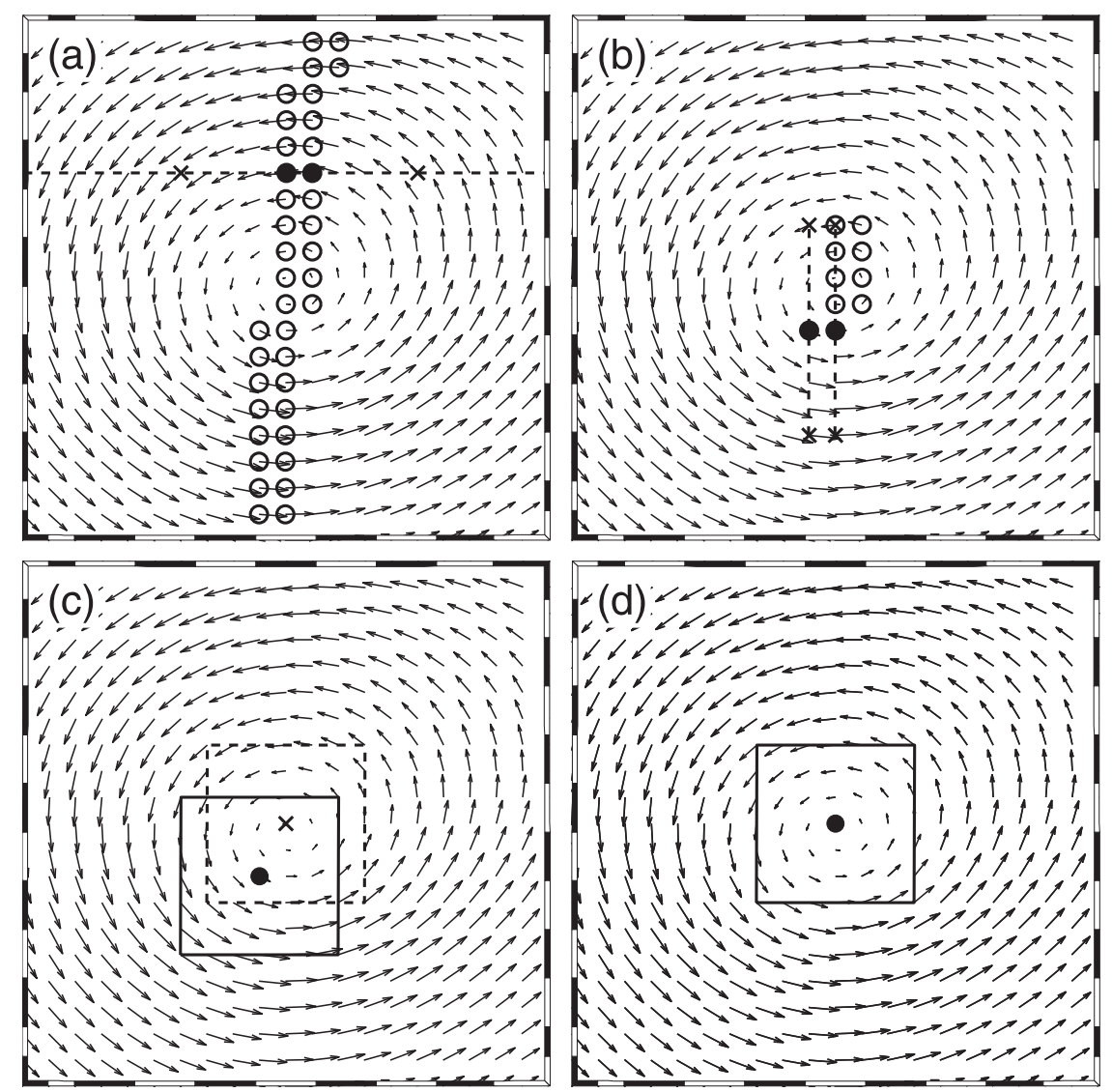

FIG. 3. (a) First, (b) second, (c) third, and (d) fourth constraint applied to the vectors within the box in the top-left corner in Fig. 2. The first constraint is applied to all latitudinal sections (the one indicated by a dashed line is used as example in the text). The second and third constraints are applied only to the points for which the previous one is satisfied. The point for which the fourth constraint is satisfied [the dot in (d)] indicates the position of an eddy center. More details are provided in the text.

latitudinal and longitudinal directions. Because $b=3$ in our case, the resulting area is $7 \times 7$ grid points. The point marked by a dot in Fig. 3c is used as an example: the solid square indicates the searching area centered around it, and the cross indicates the velocity minimum found within the area. To make sure that the minimum is local, a second search is performed within a searching area with same dimensions centered around the first minimum (dashed square). If the second minimum coincides with the first one, then it is a local minimum (as in Fig. 3c) and the point is assumed to be the possible location of an eddy center.

The velocity minima detected with the first three constraints are not necessarily associated with eddy structures and therefore cannot be automatically assumed to be eddy centers. In fact, the first three constraints can be also satisfied in regions characterized by either strong opposite currents or well-developed meanders. Both situations are represented in Fig. 4, which is an enlarged map of the region delimited by the larger box in Fig. 2. If only the first three constraints were used, then the two points indicated by the dots would be identified as eddy centers. However, the circulation around them is only partial because they are associated with a meander (the point closer to the southwest corner) and with a region of shear between opposite currents (the point closer to the northeast corner). A fourth constraint is thus needed to prevent this detection error. Overall, the first two constraints of the algorithm are used to identify the regions in the domain where local minima of velocity could represent eddy centers, and the third constraint is used to identify those minima. The fourth constraint ensures that the detected velocity minima are characterized by a closed circulation around them and thus, according to our definition, identify eddy centers.

The last constraint is applied to the velocity vectors along the boundaries of the area defined by moving $\pm(a-1)$ grid points away from the velocity minimum in 


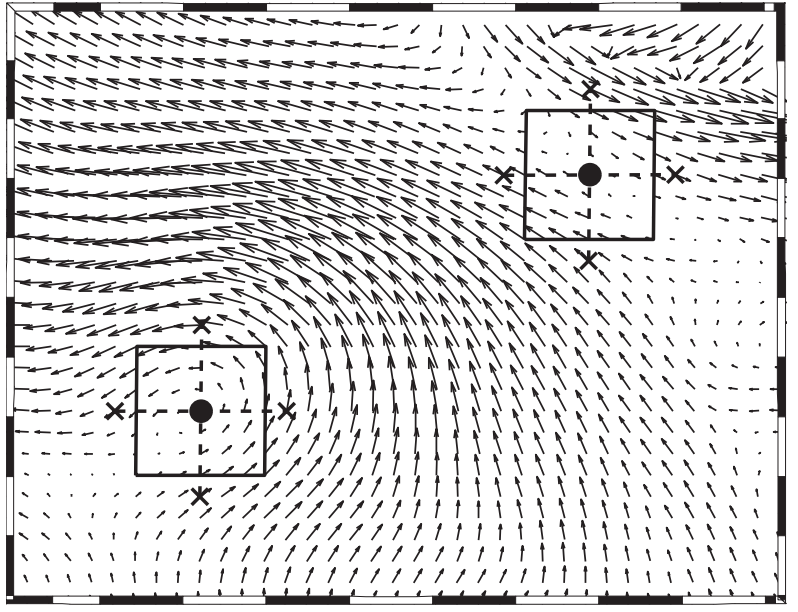

FIG. 4. Examples of points not associated with eddy structures for which the first three constraints are satisfied. The vectors along the two squares are used in Fig. 5 to describe the fourth constraint.

both directions. The resulting area for $a=4$ is $7 \times 7$ grid points. These dimensions are chosen so that the distance between the four corners of the area and the velocity minimum is close to $a$ grid points. For small values of $a$, the fourth constraint is thus applied to vectors within a similar distance from the center as the first two.

The vectors along the boundaries of the square in Fig. 3d are used to apply the fourth constraint to the velocity minimum from Fig. 3c. In Fig. 5a, the same vectors are plotted on the Cartesian plane (with the positive $x$ and positive $y$ axes oriented eastward and northward, respectively), proceeding counterclockwise from the southwest corner. This plot better illustrates the rotation of the velocity vectors around the eddy center. The sense of rotation is counterclockwise. Because of the gradual change in direction, any given vector lays either within the same quadrant as the previous one (i.e., first and second vectors in Fig. 5a) or within the next quadrant counterclockwise (i.e., fourth and third to last vectors in Fig. 5a). When this condition is satisfied by all the vectors along the boundaries of the searching area, there is a closed circulation around the velocity minimum; therefore, the point is recorded as a center of an eddy. It is important to notice that, in plots such as the one in Fig. 5a, the sense of rotation of the vectors is only dependent on the direction along which the vectors are plotted and it is independent of the sense of rotation of the eddy. Since in our case we proceed counterclockwise, vectors will rotate counterclockwise either in the presence of a cyclone or an anticyclone (the only difference between the two cases will be represented by a different orientation of the first vector).

In situations such as the ones in Fig. 4, where the local minimum of velocity is not associated with an eddy, the
Velocity vectors along searching area boundaries

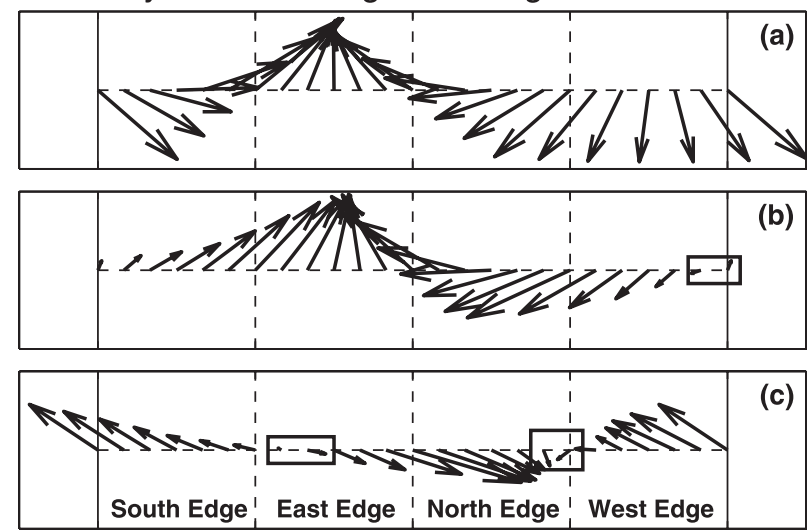

FIG. 5. (a) Vectors from the square in Fig. 3d and (b),(c) vectors from the two squares from Fig. 4 on the Cartesian plane ( $y$ direction pointing north and $x$ direction pointing east). The vectors are plotted starting from the bottom-left corner of the squares and moving counterclockwise around their edges. Vectors associated with the eddy gradually rotate counterclockwise, and the fourth constraint is respected. Vectors not associated with eddies can rotate less gradually [box in (b) and left box (c)] or can change sense of rotation [right box in (c)].

fourth constraint is not respected by all vectors. As an example, the vectors along the two squares from Fig. 4 are plotted in Fig. 5b (southwest square, representative of a well-developed meander) and Fig. 5c (northeast square, representative of a region of shear between opposite currents), similarly to Fig. 5a. The three boxes in the figures indicate vector pairs that do not respect the fourth constraint. This can occur for two distinct reasons. In the first case, evidenced by the box in Fig. $5 \mathrm{~b}$ and the left box in Fig. 5c, the directions of consecutive vectors do not lay within consecutive quadrants (i.e., they rotate from the third to the first quadrant in Fig. 5b and from the second to the fourth quadrant in Fig. 5c). In the second case, evidenced by the right box in Fig. 5c, the directions of consecutive vectors do lay within consecutive quadrants (the third and second quadrants), but the sense of rotation of the vectors has changed from counterclockwise to clockwise. Therefore, the fourth constraint can be successfully used to distinguish between local velocity minima associated with eddies and local minima that are not, reducing the excess of detection.

Once eddy centers are detected, the boundaries of each eddy are computed. In existing algorithms, these are derived using different methods, which depend on the eddy definition on which a specific algorithm is based (e.g., contours of relative vorticity or the Okubo-Weiss parameter, instantaneous streamlines). Results can significantly vary depending on the method; however, because a universal definition of eddy boundaries does not 

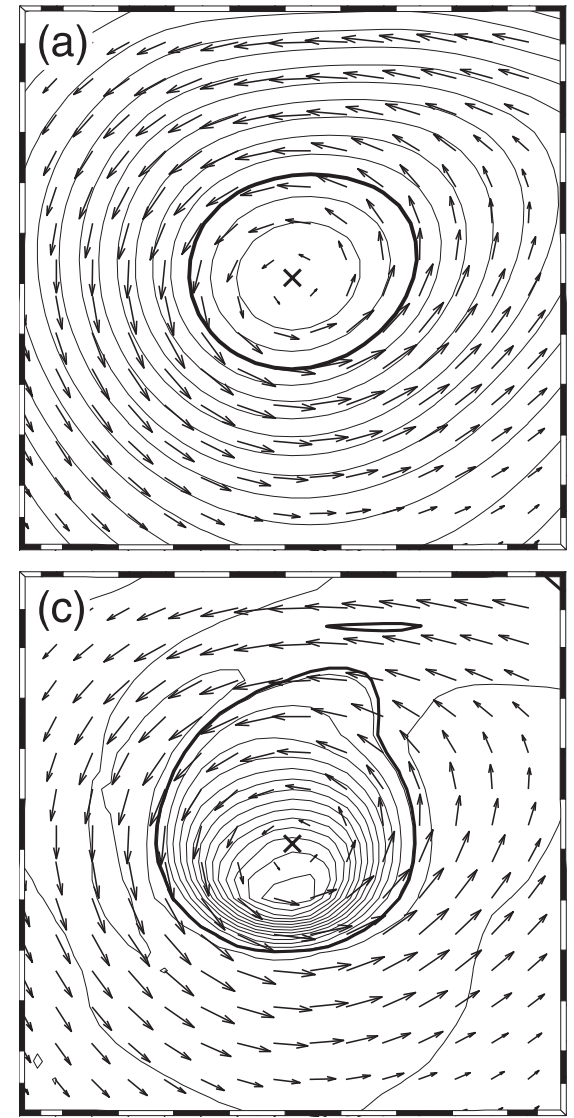

exist, each method is equally acceptable, as long as its criteria are very precisely specified and the results are tested. In our algorithm, we propose an alternative eddyedge detection procedure, in which eddy boundaries are defined from contour lines of the streamfunction field. We decided to use these contours because, to a first order, they are tangential to the velocity vectors (see Fig. 6a) because eddy velocity fields are characterized by weak divergence. A description of the method used to compute the streamfunction can be found in the appendix. Eddy boundaries are defined as the outermost closed streamline around the center, across which velocity magnitudes are still increasing in the radial direction (thicker contour in Fig. 6a). The eddy interior is therefore the region where velocity magnitude increases radially from the center. The variation of velocity across the closed contour is checked only at the four extremes of the line (i.e., the northernmost, easternmost, southernmost, and westernmost points). This represents a good compromise between algorithm efficiency and accuracy: checking the velocity variation for each point of the closed contour would drastically increase the computational requirements of the method; the four extremes are the points that are more distant from the eddy center along

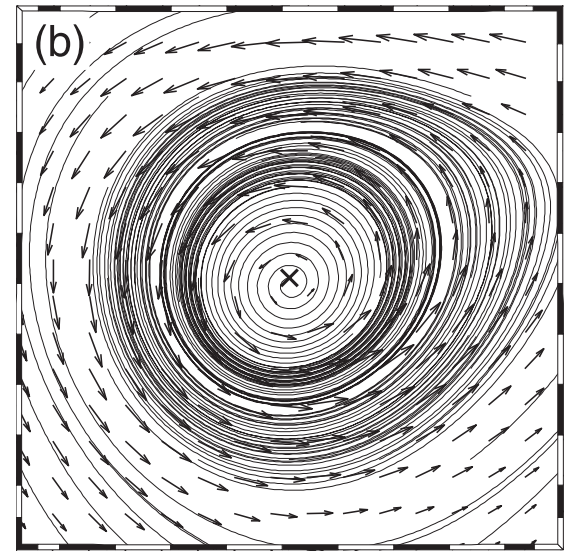

FIG. 6. (a) Contour lines of the streamfunction computed for the velocity field associated with the detected eddy from Fig. 3. The cross indicates the eddy center. The thicker line represents the eddy limits computed by the algorithm. (b) Instantaneous streamlines (analogous to the ones used for the winding-angle method) and (c) contours of the Okubo-Weiss parameter for the same velocity field. The thicker line in (c) represents the $-0.2 \sigma_{w}$ contour. In (a)-(c), vectors are plotted every two grid points.

each of the main directions, regardless of the ellipticity and orientation of the eddy, and thus, for a given contour, they are the points where a decrease in velocity magnitude is most likely to occur.

Figure 6 shows a comparison between eddy dimensions derived using our method, instantaneous streamlines, and contours of the Okubo-Weiss parameter $W$. The instantaneous streamlines in Fig. $6 \mathrm{~b}$ are analogous to the ones used in the winding-angle method by Chaigneau et al. (2008). They are different from contours of the streamfunction: they represent the trajectories of the particles advected maintaining the velocity field constant with time. Clustering the trajectories that rotate around the center would define eddy dimensions. In Fig. $6 \mathrm{c} W$ was computed using the equation introduced in section 1. Following Chaigneau et al. (2008), eddy dimensions are defined by the closed contour of $-0.2 \sigma_{w}$ (thicker line), where $\sigma_{w}$ is the spatial standard deviation of $W$. Eddy dimensions derived from our method are slightly smaller than the ones derived from instantaneous streamlines, but they are very similar to the ones obtained from the Okubo-Weiss method; eddy shape is similar in all three cases. This confirms that contours of the streamfunction can be also used to define eddy dimensions. 


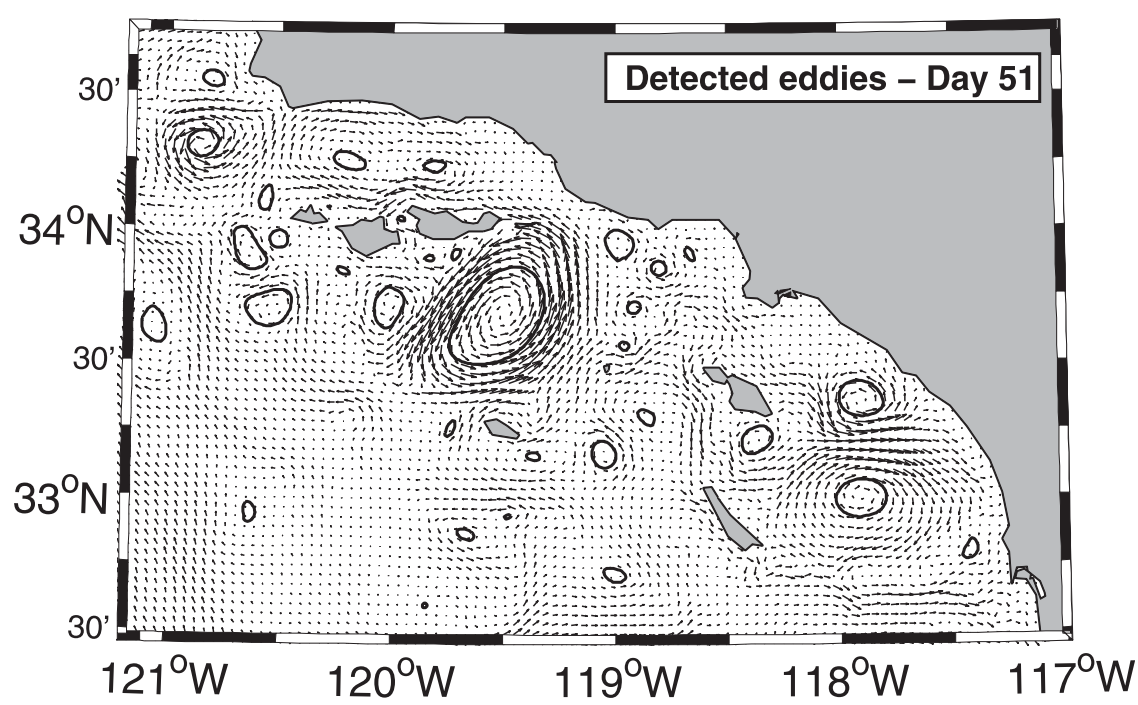

FIG. 7. Eddy field detected for day 51 (20 Feb 1996). Eddies with different shape, symmetry, and orientation can be successfully detected. Vectors are plotted every four grid points.

Because the velocity field outside the eddy can be characterized by larger divergence, the streamfunction is first computed within a small area around the eddy center. The parameter $a$ defines the minimum dimensions of detectable eddies. The area is thus defined by moving $2 a$ grid points in each direction from the eddy center. The resulting area for $a=4$ is $17 \times 17$ grid points. Because eddy size can be larger than this area, if the contour line that defines the eddy limits is too close to one of the edges (less than 1.5 grid points away, corresponding to $1.5 \mathrm{~km}$ in the SCB domain), the area is further enlarged by $a$ grid points in each direction and the streamfunction and the eddy boundaries are computed again in the larger domain. This is repeated until the computed eddy limits are far enough from the edges of the area. The streamfunction can be computed only if all the grid points within the area are ocean points. For this reason, every time land points are found within the area, its size is reduced until only ocean points are present. After this, the area will be no further increased, even if the eddy limits are close to its edges.

Velocity fields associated with small eddies can be often characterized by stronger divergence. Therefore, it is possible that some eddies have no closed contours of the streamfunction around their centers. In those cases (which represent $\approx 4.8 \%$ of the total detected eddies), eddy dimensions are assumed to be circular in shape with a radius of $a-1$ grid points ( $3 \mathrm{~km}$ in our case). This value represents the minimum distance where a closed circulation around the eddy center is observed (fourth constraint). After eddy dimensions are derived, the radius of each eddy is computed as the mean distance between the center of the eddy and all the points defining the contour line adopted as eddy limit. After eddy positions and sizes are computed, eddy trajectories are retrieved comparing successive eddy maps, as described in section $3 \mathrm{c}$.

Figure 7 shows the eddies detected by the algorithm from the velocity field from Fig. 2. Larger eddies are characterized by larger contours, confirming good accuracy and consistency of the method. Figure 8 shows contours of $-0.2 \sigma_{w}$ for the same day. The several closed contours in the figure confirm the limitations of the Okubo-Weiss method when applied to our case (specifically, high excess of detection), as already discussed in section 1. Furthermore, the figure evidences that $W$ contours are not always tangential to the velocity vectors, as it occurs for the case of the eddy in Fig. 6c. This is particularly evident in the case of the large eddy southeast of the Channel Islands and the smaller eddy within the black square (enlarged in the right panel). It is because of this undesired feature that we decided to develop an alternative method to determine eddy boundaries.

\section{b. Validation of the detection algorithm}

How to make an objective assessment of the eddies detected by an algorithm is not a trivial task (Chaigneau et al. 2008). As already discussed in the previous section, the presence of the two parameters $a$ and $b$ gives flexibility to the algorithm but also requires few sensitivity tests to choose the combination of values that will return the best results possible. Algorithm performance can be qualitatively evaluated only if the eddy distribution is known. Therefore, eddy fields automatically detected 

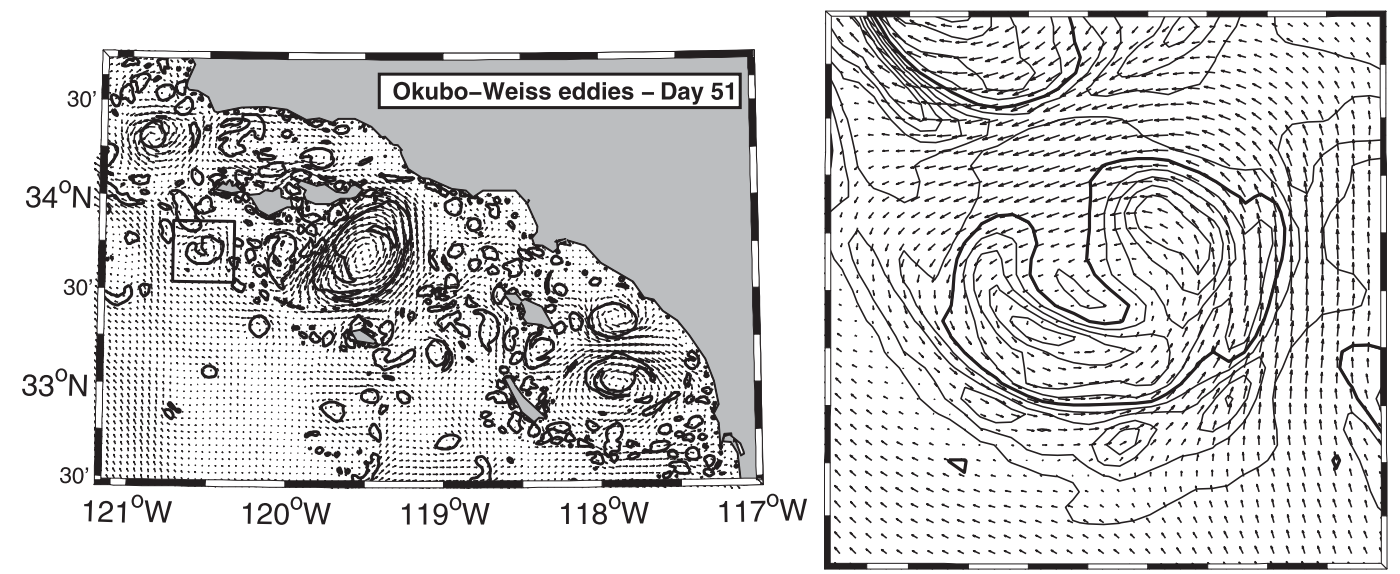

FIG. 8. (left) Contours of the Okubo-Weiss parameter $W=-0.2 \sigma_{w}$ for day 51 (20 Feb 1996). Vectors are plotted every four grid points. (right) Contours of $W$ in an enlarged map of the square from (left). All vectors are plotted. The thicker line represents the $-0.2 \sigma_{w}$ level.

for different values of $a$ and $b$ were compared to "true eddy" fields for 10 days randomly selected among the 2922 records available from the simulation. For each of these days, true eddies were manually detected from a vector map of the surface velocity field, using the eddy definition adopted in this paper as a selection criterion.

This procedure is similar to the one used in Chaigneau et al. (2008). However, in our case, true eddy maps were reconstructed directly by the authors and not derived from the results of a group of "oceanographic experts." As already evidenced in Chaigneau et al. (2008), manual eddy detection shows a wide range of results among the experts. In our case, the discrepancies among true eddy fields reconstructed by different experts (not shown) were even greater, most likely because of the dimensions and the resolution of the domain, which made it particularly challenging to identify the smaller features. For this reason, we considered the experts' results to be an unreliable scientific calibration standard.

Although larger eddies could be easily recognized in the velocity field, manual detection of the smaller eddies was facilitated by displaying in the vector maps the points for which the first three constraints of the algorithm were satisfied, setting $a=2$ and $b=1$ (minimum values for the two parameters). This made it possible to narrow down the regions in the domain where smaller eddies could have occurred, reducing the probability of not including these eddies in the true eddy fields. The presence of an eddy was determined by inspecting the velocity field on enlarged vector maps around each one of those points. Because the algorithm was developed with the intent of investigating mesoscale and submesoscale activity, eddies with a radius of two grid points (2-km radius) or smaller were removed from the true eddy maps (61 total), and they were not included in the statistical parameters used to evaluate the algorithm performance. A total of 197 true eddies were identified in the 10 maps selected for the validation. The center of each eddy was defined, consistent with our definition, as the grid point of minimum velocity. True eddy centers for days 1787 and 352 are displayed in the two vector maps in Fig. 9.

As in Chaigneau et al. (2008), the algorithm efficiency was validated defining two different parameters: the success of detection rate (SDR) and the excess of detection rate (EDR). These rates are defined as

$$
\begin{aligned}
& \mathrm{SDR}=\frac{N_{c}}{N_{\text {te }}} \times 100 \text { and } \\
& \mathrm{EDR}=\frac{N_{\text {oa }}}{N_{\text {te }}} \times 100,
\end{aligned}
$$

where $N_{\text {te }}$ (true eddies) is the total number of true eddies detected for a given day, $N_{c}$ (common) is the number of true eddies also detected by the algorithm (a true eddy is detected by the algorithm when the position of the eddy center determined by the algorithm coincides with the center position in the true eddy map), and $N_{\text {oa }}$ (only algorithm) is the number of points identified as eddy centers by the algorithm that do not correspond to a true eddy center.

The algorithm was tested for different values of the two parameters: $a$ varying from 2 to 10 and $b$ varying from 1 to 10. The SDR and EDR were computed for each different combination of the two parameters, and the results are shown in Fig. 10. As evidenced in the top panel, for a given value of $a$, the SDR decreases with increasing values of $b$. The parameter $b$ controls 

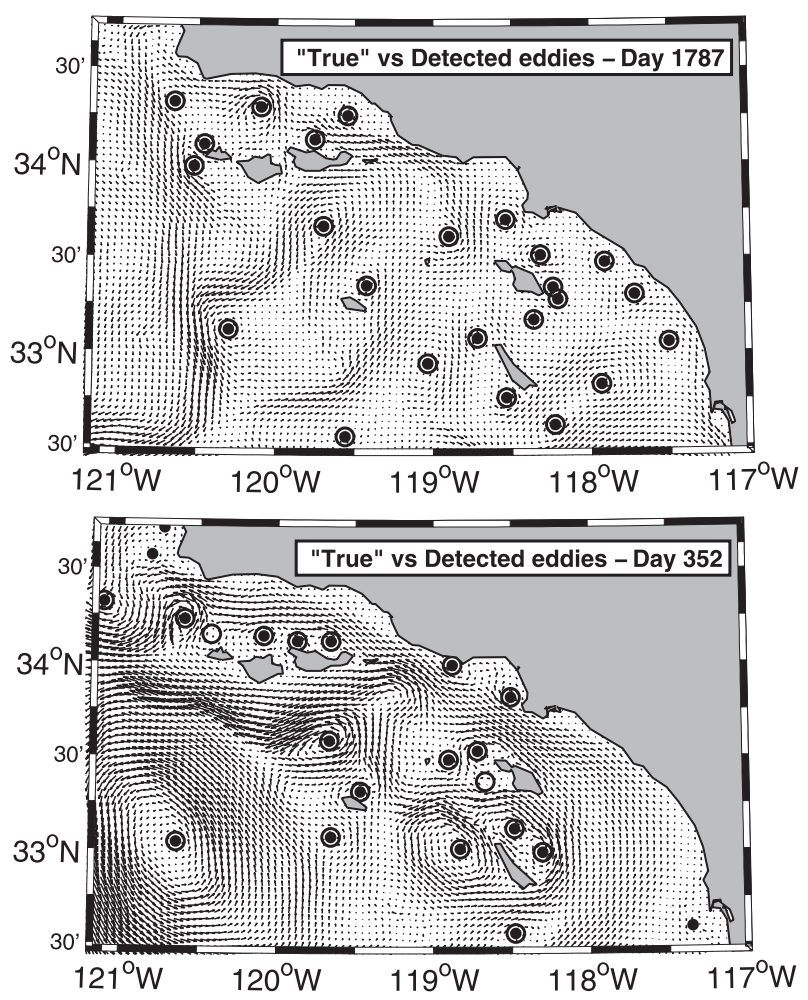

FIG. 9. Comparison between true eddies (dots) and algorithm detected eddies (circles) for days 1787 (21 Nov 2000) and 352 (17 Dec 1996). Large eddies are successfully detected. Only few smaller eddies are sometimes missed. The two wrongly detected eddies are both associated with almost closed meanders. Vectors are plotted every four grid points.

the dimension of the area within which the minimum of velocity is searched when the third constraint is applied: if the area is larger than the eddy dimensions, the top center might not satisfy the third constraint because points outside the eddy can be characterized by smaller velocity. For this reason, only larger eddies are likely to be detected for large values of $b$, thus explaining the decrease in the SDR. On the other hand, for a given value of $b$, maximum values of the SDR occur for $a$ between 3 and 4. A decrease in the SDR for large values of $a$ is somehow expected because the first two constraints will be satisfied only by the larger eddies. Reducing the value of $a$ will increase the numbers of smaller eddies that are detected. However, at the same time, the number of larger eddies that are detected can decrease because of the last constraint. In fact, larger eddies are often characterized by a broader region of small velocities around the eddy center. In such regions, the circulation around the center is sometimes not well developed, so that the rotation of the velocity vectors closest to the center might not satisfy the fourth constraint. This most likely explains the decrease in the SDR for values of $a=2$ or 3 .

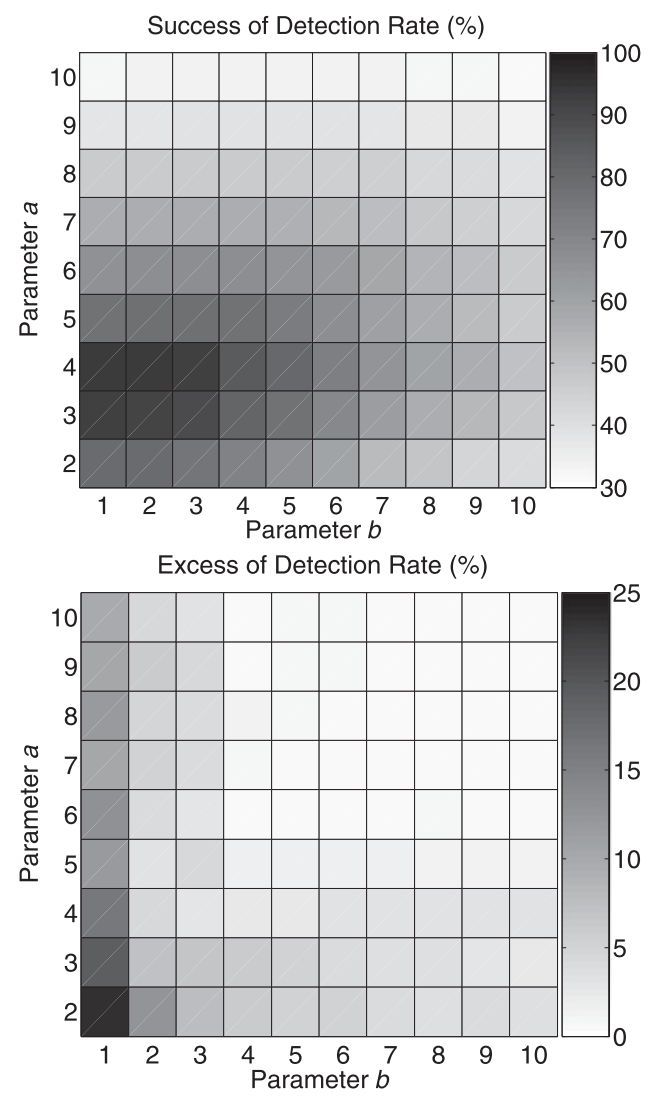

FIG. 10. SDR and EDR for different combinations of $a$ and $b$. Optimal values for eddy detection from the SCB numerical simulation are $a=4$ and $b=3$.

The bottom panel in Fig. 10 demonstrates that the algorithm is generally characterized by low EDR (below $10 \%$ ) for any combination of $a$ and $b$, except when $b=1$. In this case, the algorithm performances are affected by the small dimensions of the area used to define the velocity minimum. This occurs for two main reasons: first, a smaller searching area results in an increased number of points detected as velocity minima so that there are more points to which the fourth constraint is applied. For this reason, on a purely statistical basis, the chances to wrongly detect eddy centers are higher. Second, as can be observed from Fig. 3b, in the case of larger eddies, there are several points within an eddy for which the first two constraints are satisfied. If the searching area is not large enough, it is possible that two or more local minima of velocity are found for the same feature so that two or more centers are detected for the same eddy. Both effects result in larger EDR. It is possible that these two effects partially affect the EDR also for $b=2$ and $b=3$, especially when $a<3$. Maximum values of the SDR ( $>90 \%$ ) occur for $b$ ranging from 1 to 3 and $a=3$ and 4. The EDR within the same range of the two 
TABLE 1. Algorithm results for the 10 days used for the validation: true eddies are the eddies identified by manual detection; $N_{c}$ is the number of true eddies detected by the automated method; and $N_{\text {oa }}$ is the number of eddy centers detected by the algorithm that do not correspond to any true eddy. SDR and EDR are defined in Eqs. (1) and (2).

\begin{tabular}{lccccccccccc}
\hline \hline \multicolumn{1}{c}{ Day } & 115 & 352 & 705 & 811 & 820 & 1476 & 1626 & 1787 & 1833 & 1870 & Total \\
\hline True eddies & 19 & 20 & 15 & 20 & 16 & 18 & 16 & 24 & 26 & 23 & 197 \\
$N_{c}$ & 18 & 17 & 14 & 19 & 15 & 17 & 15 & 24 & 23 & 21 & 183 \\
$N_{\text {oa }}$ & 0 & 2 & 0 & 2 & 0 & 1 & 0 & 0 & 1 & 0 & 6 \\
Missed eddies & 1 & 3 & 1 & 1 & 1 & 1 & 1 & 0 & 3 & 2 & 14 \\
SDR (\%) & 94.7 & 85.0 & 93.3 & 95.0 & 93.7 & 94.4 & 93.8 & 100.0 & 88.5 & 91.3 & $92.9 \pm 4.0$ \\
EDR (\%) & 0.0 & 10.0 & 0.0 & 10.0 & 0.0 & 5.6 & 0.0 & 0.0 & 3.8 & 0 & $2.9 \pm 4.2$ \\
\hline
\end{tabular}

parameters is below $10 \%$ for $b=2$ or 3 and drops below $5 \%$ when $a=4$. Despite the SDR being slightly higher for $a=4$ and $b=2$, we decided that $a=4$ and $b=3$ was the optimal combination because of the lower EDR.

Table 1 summarizes the algorithm results from the 10 days used for validation, with $a=4$ and $b=3$. The average SDR is $\approx 92.9 \%$. This value is similar to the SDR observed for the winding-angle method $(\approx 92.7 \%)$ by Chaigneau et al. (2008) and higher than the SDR observed for the Okubo-Weiss method $(\approx 86.8 \%)$ in the same study. A direct comparison of the results from two studies is not possible because of the differences in the datasets to which the algorithms have been applied. Nevertheless, it is important to remark that the SDR associated with our algorithm is well above the $80 \%$ limit, which is indicated by Chaigneau et al. (2008) as the lower limit for acceptable performance by automated algorithms. Furthermore, this method is characterized by very low values of EDR, which in our case is only $\approx 2.9 \%$, whereas values from Chaigneau et al. (2008) are $\approx 18.7 \%$ for the winding-angle method and $\approx 63.3 \%$ for the Okubo-Weiss method. Such low values of the EDR are particularly important for accurate eddy tracking, as will be discussed in section $3 \mathrm{c}$. It is important to underline that these values are obtained excluding eddies with a radius of two grid points or less from the analysis. Although the EDR would not be affected, the SDR would be sensibly reduced if these small features were included.

As shown in Table 1, among the 10 days used for validation, days 1787 and 352 represent the best and the worst algorithm performances, respectively. The eddy centers detected by the algorithm for those two days are represented by the circles in the two vector maps in Fig. 9. In the same maps, true eddy centers are shown by dots. Therefore, dots surrounded by circles indicate the true eddies detected by the algorithm $N_{c}$; empty circles indicate the points wrongly detected as eddy centers $N_{\text {oa }}$; and dots without circles indicate the true eddies not detected by the algorithm (missed eddies). Missed eddies are usually small eddies that form close to the coastline or between the islands. This is expected because setting $a=4$ and $b=3$ drastically reduces the chances that all four constraints will be satisfied for eddies with radius $<4$ grid points. On the other hand, larger eddies farther away from the coast are rarely missed. As will be discussed in section $3 \mathrm{c}$, this is another characteristic that is particularly important for accurate eddy tracking. Wrongly detected eddies are most commonly induced by elongated or almost entirely detached meanders; however, as evidenced by Table 1, wrong detection does not occur frequently.

\section{c. Tracking algorithm}

Our method for eddy tracking is analogous to that proposed by Doglioli et al. (2007) and Chaigneau et al. (2008). Being a well-established procedure, we present it on this work without any formal validation. However, accuracy and performance of the tracking algorithm will be discussed in C. Dong et al. (2010, unpublished manuscript), where a complete eddy characterization of the SCB will be presented. After eddy centers are detected for the entire length of the simulation, eddy tracks are determined by comparing the centers at successive time steps, starting from day 1 . The track of a given eddy at time step $t$ is updated by searching for eddy centers of the same type (cyclonic/anticyclonic) at time $t+1$ within a searching area of $29 \times 29$ numerical grid points centered around the eddy location at time $t$.

The size of the searching area strongly affects the accuracy of the eddy tracking. In particular, to avoid splitting a continuous track into multiple tracks, eddies cannot travel outside the searching area from one time step to the successive step. For this reason, the dimension of the searching area has to be accurately defined depending on the spatial and temporal resolution of the dataset, as well as on the characteristics of the mean flow field. Because eddies are advected by the local currents, a good estimate for the searching area dimensions can be derived by multiplying the average current speed by the dataset sampling period. In our case because our dataset has a frequency of 1 day and the average current velocity within the domain is $\approx 0.2 \mathrm{~m} \mathrm{~s}^{-1}$, a searching 


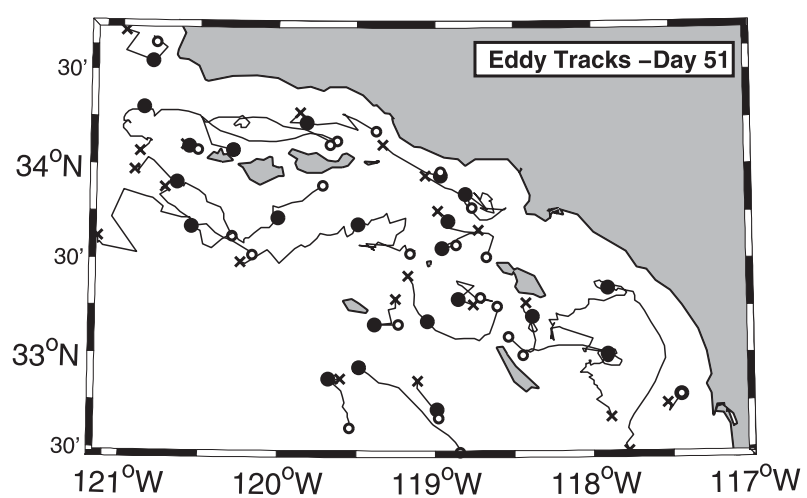

FIG. 11. Eddy tracks for the eddies detected for day 51. Empty circles and crosses represent the beginning and the end of each track, respectively. The dots represent the eddy center positions at day 51. The number of tracks is less than the number of eddies detected in Fig. 7. Some of the eddies were removed from the analysis because their lifetimes were shorter than the chosen 4-day threshold.

area with a radius of $\approx 15 \mathrm{~km}$ is large enough to prevent track splitting.

A single eddy track can be recorded as two or more distinct tracks also in case the eddy center is not detected by the algorithm for one or more days during its lifetime. This can occur for several reasons, including errors associated with the automated detection. As we have already observed, larger eddies, which are usually characterized by the longest lifetimes, are rarely missed by the algorithm. This limits the chances that a single track might be split and therefore increases the accuracy of the eddy tracking. Nevertheless, to further reduce this type of error, if a center cannot be located within the searching area at $t+1$, a second search is performed at $t+2$, with the searching area increased to $41 \times 41$ grid points. This will prevent eddy tracks from being split not only in case of a detection error but also in the eventuality that, during a particular day of its lifetime, a given eddy becomes too weak or too asymmetric to be considered an eddy.

The searching area at $t+2$ is increased only by half of its radius at $t+1$ to avoid connecting two or more distinct eddy tracks into a single track. This second type of error usually occurs if the dimensions of the searching area are too large. The fact that the detected eddies are also characterized by their sense of rotation reduces this type of error because eddy tracks are updated only if the eddy found within the searching area is of the same type. The low EDR associated with our algorithm further reduces it because there are low chances that the track of a given eddy is altered by the inclusion of wrongly detected features.

In case more than one eddy of the same type is found within the searching area, either at $t+1$ or $t+2$, the eddy track is updated with the closest center to the eddy at $t$. When no centers are detected within the searching area at $t+2$, the eddy is considered dissipated and the track for that specific eddy is closed. On the other hand, eddy centers at $t+2$ that were not connected to any eddy center at $t$ are considered newly formed eddies, and their tracks will be updated starting from time step $t+3$.

Because this algorithm was developed with the intent of detecting mesoscale and submesoscale vortices, we decided to retain in the final dataset only eddies with lifetimes that are consistent with the ones observed for these features. Submesoscale eddies are characterized by time scales on the order of a few days (Capet et al. 2008). Therefore, a lower threshold of 4 days was introduced, and all the recorded eddy tracks with a shorter lifetime were discarded: $\approx 17.1 \%$ of the tracks were characterized by a lifetime shorter than 4 days. Including this threshold in the analysis reduced the significance of the errors coming from the algorithm limitations in detecting smallscale eddies because such features are usually short lived.

Figure 11 shows the tracks for the eddies detected in Fig. 7. Only 23 tracks are plotted on the map, meaning that 10 of the 33 eddies had a lifetime shorter than 4 days. A comparison between the eddy tracks and the eddy positions and dimensions from Fig. 7 confirms that the larger eddies are associated with the longest tracks (and thus characterized by longer lifetimes), whereas the smaller eddies are the ones usually discarded because of the 4-day threshold.

\section{Eddy detection from HF radar velocities}

The application of the detection algorithm presented in this work is not limited to high-resolution numerical simulations. Being a purely geometrical method and because of the flexibility given by the two parameters $a$ and $b$, it can be used on a wide variety of velocity products: from the global fields derived from satellite products, to the regional fields obtained from lower-resolution numerical simulations, and to the coastal fields directly measured from high-frequency radar. In this section, we briefly present the preliminary results obtained by applying the algorithm to HF radar velocities from the Santa Barbara Channel for the year 2007.

To reduce the noise present in the hourly fields and to highlight the more persistent features, the HF radar velocities were averaged into daily means. For each grid point, only the mean velocities computed averaging more than six hourly records were considered significant and included in the daily fields. Grid points where velocities were not recorded or where the averages were not significant were masked as land points. The algorithm was validated for the HF radar velocities in a similar way as 


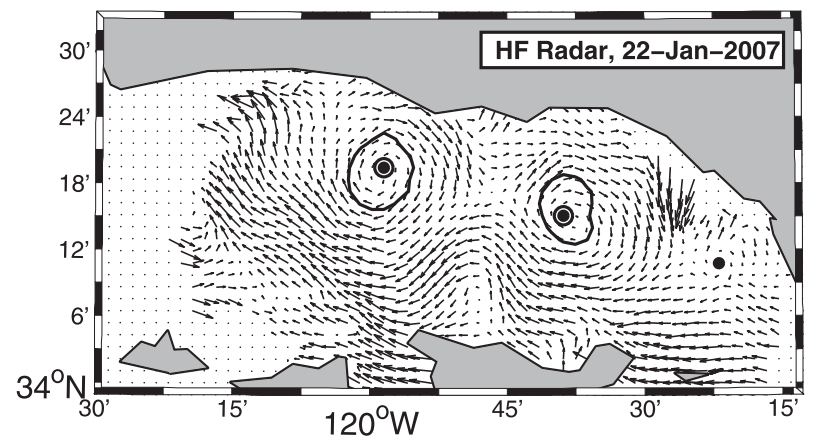

FIG. 12. Comparison between true eddies (dots) and detected eddies (circles) from HF radar surface velocities for 22 Jan 2007. The two large anticyclonic eddies are correctly detected by the algorithm. The smaller ( 1 grid point in radius) eddy in the eastern part of the domain was missed. The solid lines indicate eddy boundaries.

for the SCB numerical simulation. A total of 30 maps were randomly selected among the 365 available, and true eddy centers were manually detected for those maps. More maps were inspected for validation to have a sufficient number of eddies for the analysis of the algorithm performance. A total of 35 true eddies were identified.

Automated eddy detection was performed assigning different values to $a$ and $b$, which ranged from 2 to 10 and from 1 to 10 , respectively. The algorithm results were validated for each combination of $a$ and $b$ computing the SDR and EDR from Eqs. (1) and (2). Optimal performance (not shown) was obtained for $a=3$ and $b=1: 28$ eddies were correctly detected (SDR $=$ $80.0 \%$ ), whereas three points were wrongly detected as eddy centers $(\mathrm{EDR} \approx 8.6 \%)$. The EDR decreases for higher values of $b$ and $a$, but so does the SDR, which remains below the threshold of $80.0 \%$ for any other combination of the two parameters.

The fact that the best results are obtained for values of $a$ and $b$ lower than for the SCB case is expected because the HF radar domain is characterized by a larger grid spacing. As already observed for the SCB datasets, even in the case of HF radar velocities, larger eddies are detected with relatively high SDR, whereas eddies that are only one or two grid points in radius are characterized by a much lower SDR. Because of the coarser spatial resolution, small eddies are frequent in the HF radar dataset. On the one hand, this explains why the highest value of the SDR is obtained for $b=1$; on the other hand, this explains why it is lower than in the SCB case. The lower spatial resolution of the dataset is another reason for the low SDR because it affects the detection of larger eddies. In fact, it can occur that the center of a given eddy is located between the recorded grid points, so that the resulting velocity field is not characterized by a local velocity minimum. In this case, no grid point can satisfy the third constraint; therefore, the eddy is not detected by the algorithm. This occurred for 2 of the 35 true eddies used for validation. The EDR is relatively low, although it is higher than the one observed for the SCB. As already discussed in section $3 b$, the increase in the EDR most likely results from setting the parameter $b=1$.

As an example of the results obtained from the HF radar data, Fig. 12 shows the daily averaged surface velocity field for 22 January. As in Fig. 9, true eddies are indicated by dots and the eddies detected by the algorithm are indicated by circles. The two large eddies at the center of the domain were successfully detected by the algorithm, whereas the small eddy ( 1 grid point in radius) in the eastern part of the domain was not. The solid lines indicate the eddy boundaries as computed by the algorithm.

\section{Conclusions}

A new eddy detection algorithm based entirely on the geometry of the velocity vectors of the flow field is presented. The method was developed to investigate eddy activity in the SCB analyzing the velocity field from an 8-yr high-resolution numerical simulation using ROMS. Eddy detection is based on four constraints derived from the general characteristics of velocity fields in the presence of eddies. The constraints are applied to all the grid points in the domain, and eddy centers are recorded where all the four constraints are satisfied. Eddy boundaries around each detected center are determined by closed contours of the streamfunction field. Once eddies are detected, eddy tracks are reconstructed by connecting eddy centers of the same type found within a specified searching area at successive time steps.

The four constraints require two parameters, $a$ and $b$, to be defined. These parameters give flexibility to the algorithm, but their values need to be chosen carefully and are dependent on the spatial resolution of the dataset. To validate the algorithm and determine the values of the two parameters that maximize the SDR and minimize the EDR, the eddy fields detected by the algorithm were compared against manually detected "true eddy" fields. The validation showed that our method is reliable, with an SDR well above the lower threshold for acceptable performance by automated detection. Moreover, it is generally characterized by a very low EDR, which is particularly important to improve the accuracy of the eddy tracking. We also presented preliminary results from the application of the algorithm to HF radar surface velocities from the Santa Barbara Channel. Although the results are worse than for the SCB numerical simulation, both the SDR and the EDR remain within acceptable ranges, indicating that the method might be used to 
investigate eddy activity from this particular dataset as well.

As can be observed from Fig. 7, eddy detection is not particularly sensitive to shape, symmetry or orientation of the eddies in the domain. However, it shows some sensitivity to eddy size. In particular, eddies with a radius of only one or two grid points are characterized by lower SDR than larger eddies. This occurs mainly because in the case of small eddies the last two constraints are more difficult to be satisfied. In this respect, the algorithm performance is dependent on the spatial resolution of the dataset: 4-km radius eddies are commonly detected from the SCB velocity field because at 1-km resolution they are characterized by four gridpoint radii; however, as can be seen from Fig. 12, they can be missed when the algorithm is applied to the 2-km-resolution HF radar velocities. Because it was first developed for eddy detection from high-resolution velocity fields, further studies on lower-resolution products are needed to improve the method performance for those datasets. Still maintaining the general concepts presented in this paper, this might require a few minor modifications and refinements of the algorithm. Preliminary results from the analysis of the eddy statistics derived for the entire length of the numerical simulation (not shown) indicate that the tracking method as well as the method developed to derive eddy dimensions may still need further refinement.

Future work will include the application of this method to different velocity fields, ranging from regional to global and derived from both remotely sensed quantities as well as numerical model simulations [the algorithm is currently tested on velocity fields derived from satellite SLA and SST, as well as on global velocities from the Estimating the Circulation and Climate of the Ocean, Phase II (ECCO2) dataset]. From a more general point of view, all the existing automated detection methods are characterized by good SDR and relatively low EDR. Nonetheless, all the methods, including the one presented in this work, have specific limitations. A direct comparison among these methods on common datasets could be extremely beneficial to further improve automated eddy detection, and it might lead to a new common algorithm in which the strengths of each individual method can be enhanced and their respective limitations can be minimized.

Acknowledgments. The authors want to thank T. Chaffey, J. Watson, and E. Idica for their help with the algorithm validation. A special thanks to Eileen Idica for providing the scripts to process HF radar velocities. Support for FN and TD was provided under Dickey's Office of Naval Research, Secretary of the Navy/Chief of Naval Operation Chair in Oceanographic Science. CD appreciates supports from the National Science Foundation (OCE 06-23011) and the National Aeronautics and Space Administration (Grant NNX08AI84G).

\section{APPENDIX}

\section{Streamfunction Computation}

Assuming $\psi(1,1)=0$, the streamfunction at a given point $(i, j)$ is computed as

$$
\psi(i, j)=\frac{\left(\psi_{x y}+\psi_{y x}\right)}{2},
$$

where

$$
\begin{aligned}
& \psi_{x y}=-\sum_{x=1}^{i} v(x, 1) \Delta x+\sum_{y=1}^{j} u(i, y) \Delta y \quad \text { and } \\
& \psi_{y x}=-\sum_{x=1}^{i} v(x, j) \Delta x+\sum_{y=1}^{j} u(1, y) \Delta y .
\end{aligned}
$$

Here, $u$ and $v$ are the two components of velocity and $\Delta x$ and $\Delta y$ are the longitudinal and latitudinal grid spacings, respectively. The sums in Eqs. (A2) and (A3) are trapezoidal sums.

Both $\psi_{x y}$ and $\psi_{y x}$ are discretized forms of the integral

$$
\int_{(1,1)}^{(i, j)} \psi=\int_{(1,1)}^{(i, j)}(-v d x+u d y) \text {. }
$$

The difference between the two is the path of integration: $\psi_{x y}$ is computed by first integrating $v$ along the $x$ direction at $y=1$ and then $u$ along the $y$ direction at $x=i$, whereas $\psi_{y x}$ is computed by first integrating $u$ along the $y$ direction at $x=1$ and then $v$ along the $x$ direction at $y=j$. Because the velocity filed is never divergent free, the value of $\psi$ is dependent on the path of integration, and thus $\psi_{x y}$ is slightly different than $\psi_{y x}$. By taking the average of the two quantities in Eq. (A1), we reduce this error so that that the resulting contours of $\psi$ are as close as possible to be velocity streamlines.

\section{REFERENCES}

Benitez-Nelson, C. R., and Coauthors, 2007: Mesoscale eddies drive increased silica export in the subtropical Pacific Ocean. Science, 316, 1017-1021.

Caldeira, R. M. A., P. Marchesiello, N. P. Nezlin, P. M. DiGiacomo, and J. C. McWilliams, 2005: Island wakes in the Southern California Bight. J. Geophys. Res., 110, C11012, doi:10.1029/ 2004JC002675. 
Capet, X., J. C. McWilliams, M. J. Molemaker, and A. F. Shchepetkin, 2008: Mesoscale to submesoscale transition in the California Current system. Part I: Flow structure, eddy flux, and observational tests. J. Phys. Oceanogr., 38, 29-43.

Carton, J. A., G. Chepurin, X. Cao, and B. Giese, 2000: A Simple Ocean Data Assimilation analysis of the global upper ocean 1950-95. Part I: Methodology. J. Phys. Oceanogr., 30, 294-309.

Chaigneau, A., A. Gizolme, and C. Grados, 2008: Mesoscale eddies off Peru in altimeter records: Identification algorithms and eddy spatio-temporal patterns. Prog. Oceanogr., 79 (2-4), 106-119.

Chelton, D. B., M. G. Schlax, R. M. Samelson, and R. A. de Szoeke, 2007: Global observations of large oceanic eddies. Geophys. Res. Lett., 34, L15606, doi:10.1029/2007GL030812.

Conil, S., and A. Hall, 2006: Local regimes of atmospheric variability: A case study of Southern California. J. Climate, 19, 4308-4325.

Dickey, T. D., F. Nencioli, V. S. Kuwahara, C. Leonard, W. Black, Y. M. Rii, R. R. Bidigare, and Q. Zhang, 2008: Physical and bio-optical observations of oceanic cyclones west of the island of Hawai'i. Deep Sea Res. II, 55 (10-13), 1195-1217.

Doglioli, A. M., B. Blanke, S. Speich, and G. Lapeyre, 2007: Tracking coherent structures in a regional ocean model with wavelet analysis: Application to Cape Basin eddies. J. Geophys. Res., 112, C05043, doi:10.1029/2006JC003952.

Dong, C., and J. C. McWilliams, 2007: A numerical study of island wakes in the Southern California Bight. Cont. Shelf Res., 27, 1233-1248.

_ - E. Y. Idica, and J. C. McWilliams, 2009a: Circulation and multiple-scale variability in the Southern California Bight. Prog. Oceanogr., 82, 168-190.

_ side of Lanai Island, Hawai'i. J. Geophys. Res., 114, C10008, doi:10.1029/2009JC005346.

Emery, B., L. Washburn, and J. Harlan, 2004: Evaluating radial current measurements from CODAR high-frequency radars with moored current meters. J. Atmos. Oceanic Technol., 21, 1259-1271.

Falkowski, P. G., D. Ziemann, Z. Kolber, and P. K. Bienfang, 1991: Role of eddy pumping in enhancing primary production in the ocean. Nature, 352, 55-58.
Isern-Fontanet, J., E. Garcia-Ladona, and J. Font, 2003: Identification of marine eddies from altimetric maps. J. Atmos. Oceanic Technol., 20, 772-778.

McGillicuddy, D. J., Jr., and Coauthors, 1998: Influence of mesoscale eddies on new production in the Sargasso Sea. Nature, 394, 263-266.

McNeil, J. D., H. W. Jannasch, T. Dickey, D. McGillicuddy, M. Brzezinski, and C. M. Sakamoto, 1999: New chemical, bio-optical and physical observations of upper ocean response to the passage of a mesoscale eddy off Bermuda. J. Geophys. Res., 104 (C7), 15 537-15 548.

McWilliams, J. C., 1990: The vortices of two-dimensional turbulence. J. Fluid Mech., 219, 361-385.

2008: The nature and consequence of oceanic eddies. Ocean Modeling in an Eddying Regime, Geophys. Monogr., Vol. 177, Amer. Geophys. Union, 5-15.

Morrow, R., F. Birol, D. Griffin, and J. Sudre, 2004: Divergent pathways of cyclonic and anti-cyclonic ocean eddies. Geophys. Res. Lett., 31, L24311, doi:10.1029/2004GL020974.

Nencioli, F., V. S. Kuwahara, T. D. Dickey, Y. M. Rii, and R. R. Bidigare, 2008: Physical dynamics and biological implications of a mesoscale eddy in the lee of Hawai'i: Cyclone Opal observations during E-Flux III. Deep Sea Res. II, 55 (10-13), $1252-1274$.

Okubo, A., 1970: Horizontal dispersion of floatable particles in vicinity of velocity singularities such as convergences. DeepSea Res., 17, 445-454.

Olson, D. B., 1991: Rings in the ocean. Annu. Rev. Earth Planet. Sci., 19, 283-311.

Robinson, S. K., 1991: Coherent motions in the turbulent boundarylayer. Annu. Rev. Fluid Mech., 23, 601-639.

Sadarjoen, I. A., and F. H. Post, 2000: Detection, quantification, and tracking of vortices using streamline geometry. Comput. Graphics, 24, 333-341.

Shchepetkin, A. F., and J. C. McWilliams, 2005: The regional oceanic modeling system (ROMS): A split-explicit, free-surface, topography-following-coordinate oceanic model. Ocean Modell., 9, 347-404.

Weiss, J., 1991: The dynamics of enstrophy transfer in 2-dimensional hydrodynamics. Physica D, 48 (2-3), 273-294. 